\title{
RESEARCH NOTES
}

\section{NOTE ON HÖLDER INEQUALITIES}

\author{
SUNG GUEN KIM \\ Department of Mathematics \\ Pohang Institute of Science \& Technology \\ P.O. Box 215, Pohang 790-600, Korea
}

(Received April 13, 1992 and in revised form June 26, 1993)

ABSTRACT. In this note, we show that if $m, n$ are positive integers and $x_{\mathfrak{t}} \geq 0$, for $i=1, \cdots, n$, for $j=1, \cdots, m$, then

$$
\left(\sum_{i=1}^{n} x_{i 1} \cdots x_{\imath m}\right)^{m} \leq\left(\sum_{i=1}^{n} x_{\imath 1}{ }^{m}\right) \cdots\left(\sum_{i=1}^{n} x_{\imath m}{ }^{m}\right)
$$

with equality, in case $\left(x_{11}, \cdots, x_{n 1}\right) \neq 0$ if and only if each vector $\left(x_{1}, \cdots, x_{n}\right), j=1, \cdots, m$, is a scalar multiple of $\left(x_{11}, \cdots, x_{n 1}\right)$. The proof is a straight-forward application of Hölder inequalities. Conversely, we show that Hölder inequalities can be derived from the above result.

KEY WORDS AND PHRASES. The Hölder Inequalities.

1991 AMS SUBJECT CLASSIFICATION CODES. 26D15.

\section{MAIN RESULTS.}

LEMMA 1. If $m, n$ are positive integers and $x_{i j} \geq 0$, for $i=1, \cdots, n$, for $j=1, \cdots, n$, then

$$
\left(\sum_{i=1}^{n} x_{i 1} \cdots x_{\imath m}\right)^{m} \leq\left(\sum_{\imath=1}^{n} x_{i 1}{ }^{m}\right) \cdots\left(\sum_{i=1}^{n} x_{t m}{ }^{m}\right)
$$

with equality, in case $\left(x_{11}, \cdots, x_{n 1}\right) \neq 0$ if and only if each vector $\left(x_{1}, \cdots, x_{n \jmath}\right), j=1, \cdots, m$, is a scalar multiple of $\left(x_{11}, \cdots, x_{n 1}\right)$.

PROOF. Use induction on $m$. When $m=1$, the above inequalities are trivial. Suppose that the above inequalities hold with $m-1$. Then it follows that

$$
\begin{aligned}
& \left(\sum_{i=1}^{n} x_{i 1} \cdots x_{\imath m}\right) \leq\left\{\sum_{i=1}^{n}\left(x_{i 1} \cdots x_{i m-1}\right)^{\frac{m}{m-1}}\right\}^{\frac{m-1}{m}} \cdot\left\{\sum_{i=1}^{n} x_{i m}^{m}\right\}^{\frac{1}{m}}, \quad \text { (by Hölder Inequalities) } \\
& =\left\{\sum_{i=1}^{n} x_{\imath 1}^{\frac{m}{m-1}} \cdots x_{\imath m-1}{ }^{\frac{m}{m-1}}\right\}^{\frac{m-1}{m}} \cdot\left\{\sum_{i=1}^{n} x_{\imath m}^{m}\right\}^{\frac{1}{m}} \\
& \leq\left\{\sum_{i=1}^{n} x_{i 1}^{\frac{m}{m-1} \cdot(m-1)} \cdots \sum_{i=1}^{n} x_{i m-1^{\frac{m}{m-1}} \cdot(m-1)}\right\}^{\frac{1}{m}} \cdot\left\{\sum_{i=1}^{n} x_{t_{m}}\right\}^{\frac{1}{m}}, \text { (by Induction Hypothesis) } \\
& =\left\{\sum_{i=1}^{n} x_{\imath 1}{ }^{m} \cdots \sum_{i=1}^{n} x_{\imath m-1}{ }^{m} \cdot \sum_{i=1}^{n} x_{\imath m}{ }^{m}\right\}^{\frac{1}{m}}
\end{aligned}
$$

Therefore the proof is complete. 
Note that the above inequalities have been deduced using Hölder Inequalities. We can also deduce Hölder Inequalities by using the above inequalities.

THEOREM 1. Given $p_{1}, \cdots, p_{n} \in R$ with $p_{k}>1$, for each $k=1, \cdots, n$ and $\Sigma_{k=1}^{n} \frac{1}{p_{k}}=1$ and given $a_{1}, \cdots, a_{n}>0$, we have the following inequality

$$
a_{1} \cdots a_{n} \leq \sum_{k=1}^{n} \frac{a_{k}^{p k}}{p_{k}}
$$

PROOF. First we prove this theorem when all $p_{k}$ 's are rational. Write $p_{k}=\frac{c_{k}}{b_{k}}$ for some $b_{k}, c_{k} \in N$ for $1 \leq k \leq n$. Let $m_{1}=2 \cdot 1 \mathrm{~cm}\left(c_{1}, \cdots, c_{n}\right)$. Let $q_{k}=\frac{m}{p_{k}}$ for $1 \leq k \leq n$. It is clear that $q_{k} \geq 2$ for $1 \leq k \leq n$. Let $x_{k}=a_{k}^{\frac{1}{q_{k}}}$ for $1 \leq k \leq n$. Let $S: R^{m} \rightarrow R^{m}$ be the mapping defined by

$$
S\left(y_{1}, y_{2}, \cdots, y_{m}\right)=\left(y_{m}, y_{1}, y_{2}, \cdots, y_{m-1}\right)
$$

for $\left(y_{1}, y_{2}, \cdots, y_{m}\right) \in R^{m}$. Define $m$ vectors $Z_{1}, \cdots, Z_{m}$ by

$$
Z_{1}=(\underbrace{q_{1}-\text { times }}_{x_{1}, \cdots, x_{1}}, \underbrace{q_{2}-\text { times }}_{x_{2}, \cdots, x_{2},} \cdots, \underbrace{q_{m}-\text { times }}_{x_{m}, \cdots, x_{m}})
$$

and $Z_{i}=S\left(Z_{i_{-1}}\right)$ for $2 \leq i \leq m$. Applying the Lemma 1 to the $m$ vectors $Z_{1}, \cdots, Z_{m}$, we have

$$
m \cdot x_{1}^{q_{1}} \cdots x_{n}^{q_{n}} \leq q_{1} \cdot x_{1}{ }^{m}+\cdots+q_{n} \cdot x_{n}{ }^{m}
$$

and equality holds if and only if $x_{1}=x_{k}$ for $2 \leq k \leq n$.

By substituting $x_{k}^{m}=a_{k}^{p_{k}}(1 \leq k \leq n)$ into both sides in (1.1), we have

$$
a_{1} \cdots a_{n} \leq \sum_{k=1}^{n} \frac{a_{k}^{p_{k}}}{p_{k}},
$$

and equality holds if and only if $a_{1}^{p_{1}}=a_{k}^{p_{k}}$ for $2 \leq k \leq n$. Now, let us show the theorem when all $p_{k}$ 's are real. We can choose $n$ sequences of rational numbers $\left\{r_{1},\right\}, \cdots,\left\{r_{n},\right\}$ satisfying $r_{k},>1$ for $1 \leq k \leq n$, all $j \in N$ and $\Sigma_{k=1}^{n} \frac{1}{r_{k}}=1$ for each $j \in N$ and $r_{k} \rightarrow p_{k}$ as $j \rightarrow \infty$, for $1 \leq k \leq n$. By the above argument, for each $j \in N$, we have

$$
a_{1} \cdots a_{n} \leq \sum_{i=1}^{n} \frac{a_{k}^{p_{k}}}{r_{k j}}
$$

Taking the limit as $j \rightarrow \infty$, the result follows.

Hölder Inequalities follow from Theorem 1 in the usual way, that can be found in most text books. From Lemma 1 and Theorem 1, we know that the following form of inequalities is essential for the Hölder inequalities: If $n$ is a positive integer and $x_{i}, \geq 0$, for $i=1, \cdots, n$, for $j=1, \cdots, n$, then

$$
\left(\sum_{i=1}^{n} x_{i 1} \cdots x_{i n}\right)^{n} \leq\left(\sum_{i=1}^{n} x_{i 1}{ }^{n}\right) \cdots\left(\sum_{i=1}^{n} x_{i n}{ }^{n}\right) .
$$

ACKNOWLEDGEMENT. This work was partially funded by a grant from the Garg-Kosef. 


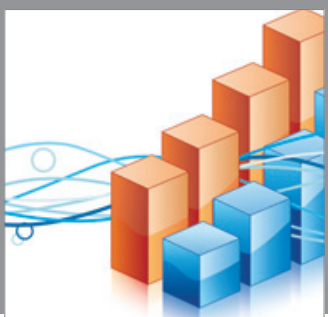

Advances in

Operations Research

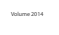

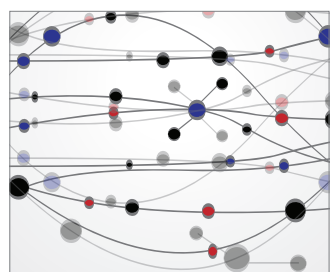

\section{The Scientific} World Journal
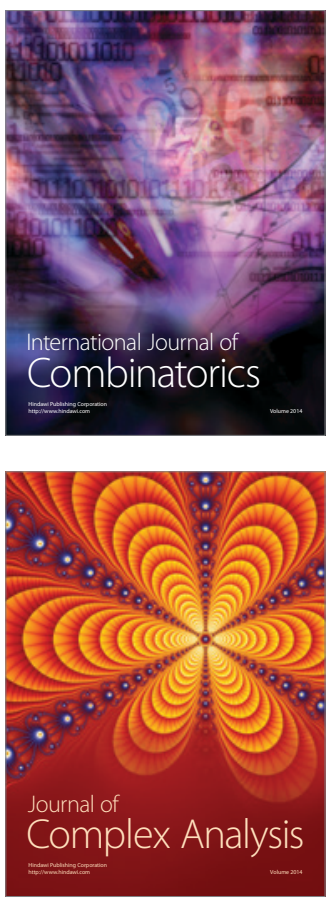

International Journal of

Mathematics and

Mathematical

Sciences
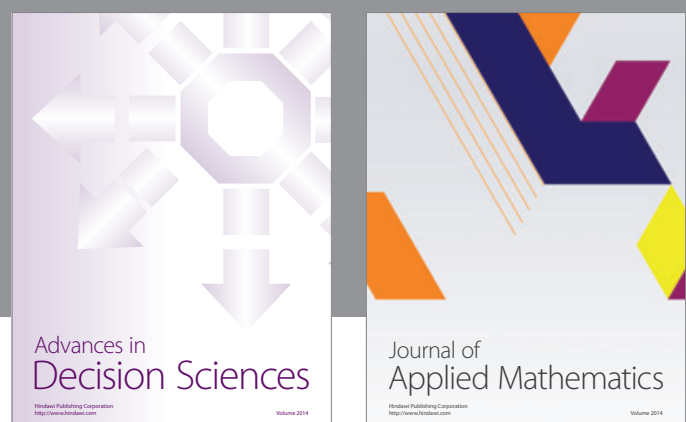

Journal of

Applied Mathematics
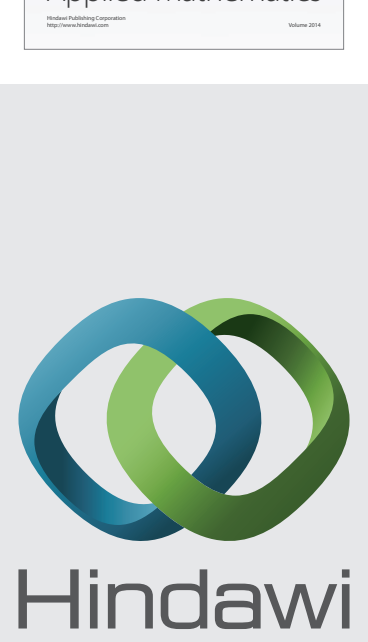

Submit your manuscripts at http://www.hindawi.com
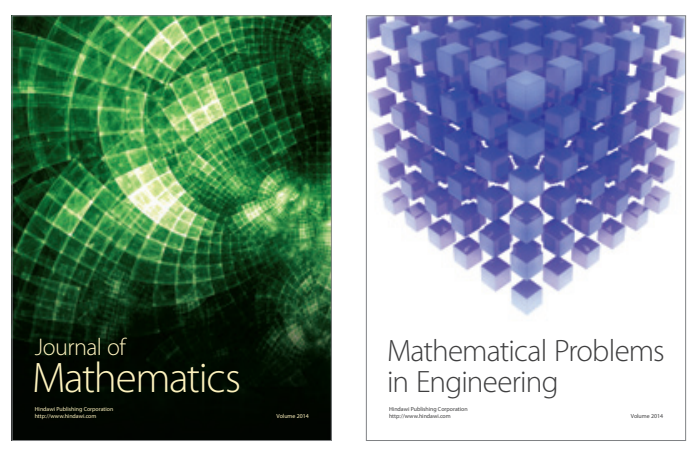

Mathematical Problems in Engineering
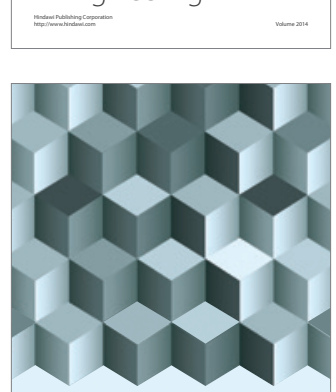

Journal of

Function Spaces
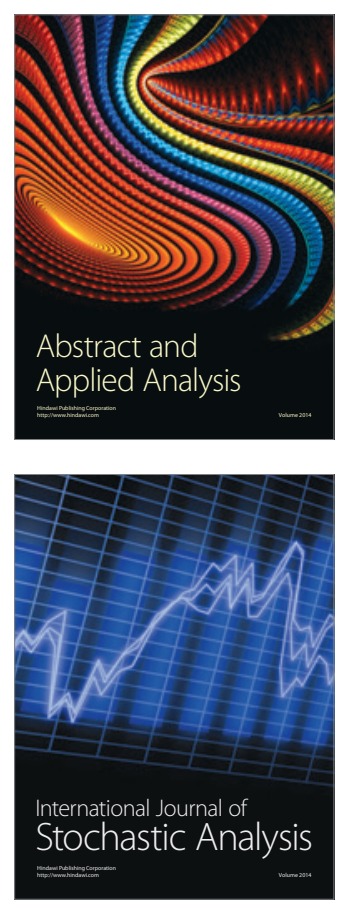

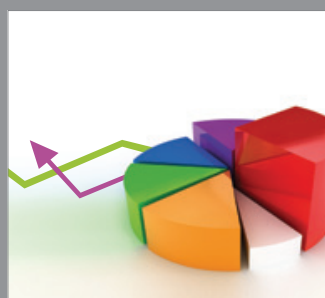

ournal of

Probability and Statistics

Promensencen
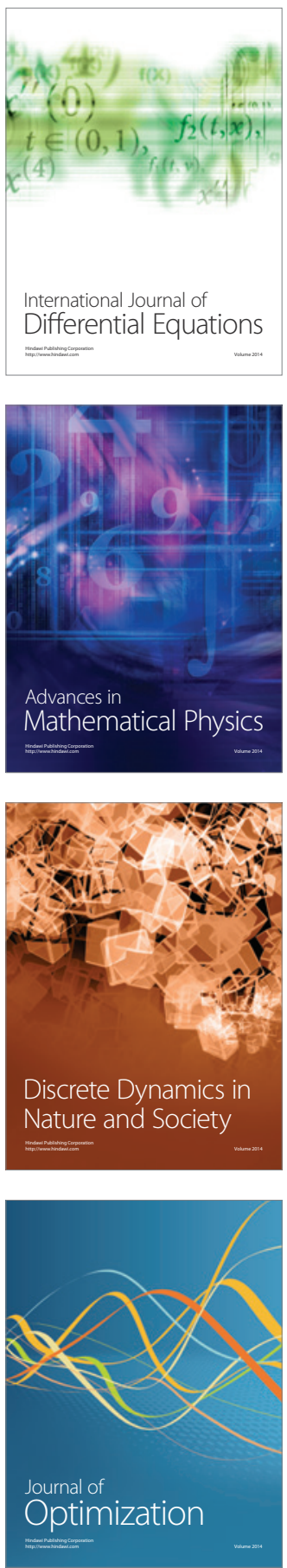